\title{
Numerical Simulation of Thermal Signatures of Buried Mines over a Diurnal Cycle
}

\author{
İbrahim Kürşat Şendur and Brian A. Baertlein \\ The Ohio State University, ElectroScience Laboratory \\ 1320 Kinnear Road, Columbus, OH 43212
}

\begin{abstract}
Three-dimensional thermal and radiometric models have been developed to study the passive IR signature of a land mine buried under a rough soil surface. A finite element model is used to describe the thermal phenomena, including temporal variations, the spatial structure of the signature, and enviromental effects. The Crank-Nicholson algorithm is used for time-stepping the simulation. The mine and the surroundings are approximated by pentahedral elements having linear interpolation functions. The FEM grid for the soil includes a random rough surface having a normal probability density and specified covariance function. The mine is modeled as a homogeneous body of deterministic shape having the thermal properties of TNT. Natural solar insolation (both direct and atmosphericscattered components) and the effects of convective heat transfer are represented by linearized boundary conditions. The behavior over a periodic diurnal cycle is studied by running the simulation to steady state. Finite element solutions for the thermal emissions are combined with reflected radiometric components to predict the signatures seen by an IR camera. Numerical simulations are presented for a representative target, a $25 \mathrm{~cm}$ anti-tank mine simulant developed by the US Army. The temporal evolution of the temperature distribution and IR signature are presented for both smooth and rough surfaces.
\end{abstract}

Keywords: Thermal infrared imagery, land mines, finite element method, heat transfer, radiometry, surface roughness, thermal model, numerical simulation

\section{INTRODUCTION}

In this paper three-dimensional thermal and radiometric models are developed for soil containing a buried land mine. The objective of this work is to improve our understanding of the physical processes that produce the thermal mine signature. It is our hope that this model will also improve comprehension and interpretation of thermal IR imagery, suggest more efficient usage of IR sensors, and lead to the development of more robust signal processing techniques.

IR detection of land mines has a long history, and recently there has been extensive work in this area. Most existing studies, however, are concerned with signal processing algorithms or the performance achieved by a particular sensor and not with the underlying physical bases of the signatures. Although many experimental and empirical studies of these signatures have been performed, the underlying phenomena are still poorly understod. Prior studies and anecdotal evidence have implicated several physical phenomena including thermal effects related to the mine, soil disturbances, and changes in soil moisture. The presence of the mine also indirectly influences the vitality of overlying vegetation, which may have a role in the signature. Among the physics-related papers on thermal imaging we note the work of LeSchack and Del Grande ${ }^{1}$ who describe the effects of emissivity on target signatures in the context of a two-color IR system. Janssen et al. ${ }^{2}$ reported a study of basic phenomenology in which several sensors were used to examine the time evolution of signatures for both buried and surface mines. A related study is that of Maksymomko et al. ${ }^{3}$ who measured the temperatures of both live and surrogate mines through multiple diurnal cycles. Simard ${ }^{4}$ found a linear relation between the apparent temperature of buried mines and the soil temperature gradient on gravel roads. Russel et al. ${ }^{5}$ showed that this gradient could be predicted from remote soil temperature measurements, making it possible to assess the potential effectiveness of an IR sensor prior to use. The possibility of using polarization information was examined briefly by Barbour et al. ${ }^{6}$ and Larive et al. ${ }^{7}$ DiMarzio et al. ${ }^{8}$ present spectroscopic data collected by a non-imaging sensor. Pregowski and Swiderski ${ }^{9}$ present a qualitative discussion of the effects of soil cover and water content. Another work, obliquely related to the effort proposed here, is that of Del Grande et al., ${ }^{10}$ who discuss the use of computed tomography and a finite difference thermal analysis code

Corresponding author: B.A.B. (614) 292-0076 (voice), (614) 292-7297 (fax), baertlein.1@osu.edu 
(TOPAZ3D) for imaging structural flaws in materials. Discussions of the physics of non-thermal hyperspectral sensors are rare. Among these works we note a paper by DePersia et al. ${ }^{11}$ in which the spectral signatures of various types of soil disturbances are discussed. A simulation tool for surface mines in the UV to near IR is described by Flynn et al. ${ }^{12}$

While models for a mine's thermal signature are not well developed, there has been progress in modeling the temperature of homogeneous soil over a diurnal cycle. ${ }^{13-18}$ In a previous unpublished work ${ }^{19}$ the authors extended those efforts to develop one-dimensional models for natural solar heating of soil and mines. Those models address both homogeneous earth and layered earth containing strata with mine-like properties. We developed analytical models to predict the temperature distribution on the surface and at depth. In spite of the crude approximations inherent in a 1-D model, the results predict many phenomena observed in thermal mine imagery, including the presence of cross-over times, the time lag between solar flux and mine signatures, signature differences between thermally conducting and insulating mines, and the effects of depth on IR detection.

This work begins with a discussion of the underlying physics in Section 2. The mathematical formulation of the thermal model is given in Section 3, which also presents the FEM formulation and assumptions regarding the boundary conditions (whereby the source is introduced). The radiometric models are discussed in Section 4. Models for the sunlight and skylight, the rough surface approximation for the soil surface, and the radiometric quantities are discussed. Simulation results for a representative simulant anti-tank mine are presented in Section 5. Concluding remarks appear in Section 6.

\section{PHYSICAL DESCRIPTION OF PROCESSES}

Thermal emission from the soil over a buried mine depends on the surface temperature and emissivity. The surface temperature is a result of (1) the solar-driven, diurnal flow of thermal energy into and out of the soil, (2) the effect of the buried mine on that flow, and (3) IR radiation from the surface. Three heat transfer mechanisms are involved in these processes. Conduction occurs in the soil and at the soil-mine interface. At the soil-air interface convective and radiative mechanisms are present. Convection involves the transfer of heat between the soil and air and is greatly affected by wind. Radiative heat transfer involves direct solar radiation, atmosphere-scattered solar radiation, and thermal radiation between the earth and atmosphere. All of these processes are time varying (and herein, assumed periodic) over the diurnal cycle. In this work we measure time $t$ with respect to midnight local time. Mathematical descriptions of the phenomena are as follows:

The temperature distribution in the soil and mine is described by the three-dimensional heat flow equation

$$
\rho(\mathbf{r}) C_{p}(\mathbf{r}) \frac{\partial T(\mathbf{r}, t)}{\partial t}=\nabla \cdot(\mathcal{K}(\mathbf{r}) \nabla T(\mathbf{r}, t)),
$$

where $T(\mathbf{r}, t)[\mathrm{K}]$ is the temperature distribution, $\mathcal{K}\left[\mathrm{W} \mathrm{m}^{-1} \mathrm{~K}^{-1}\right]$ is the thermal conductivity of the material, $C_{p}[\mathrm{~J}$ $\left.\mathrm{kg}^{-1} \mathrm{~K}^{-1}\right]$ is the specific heat at constant pressure and $\rho\left[\mathrm{kg} / \mathrm{m}^{3}\right]$ is the density of the material. Convective and radiative heat transfer drive the thermal flow and are introduced via a boundary condition at the soil-air interface. Prior one-dimensional analyses of the problem have addressed this topic and are relevant here. ${ }^{13-15,17,18,20}$ We write

$$
\left.\mathcal{F}_{\text {net }}(t)\right|_{\text {interface }}=\mathcal{F}_{\text {sun }}(t)+\mathcal{F}_{\text {sky }}(t)-\mathcal{F}_{\text {sh }}(t)-\mathcal{F}_{\text {gr }}(t),
$$

where $\mathcal{F}_{\text {net }}$ is the net heat flux into the ground, $\mathcal{F}_{\text {sun }}$ is the incident solar radiation reduced by cloud extinction, atmospheric absorption, soil albedo and the cosine of the zenith angle, $\mathcal{F}_{s k y}$ is the sky brightness with a correction for cloud cover, $\mathcal{F}_{s h}$ is the sensible heat transfer from land to atmosphere due to convection, and $\mathcal{F}_{g r}$ is the gray body emission from soil's surface.* Watson ${ }^{13}$ used

$$
\mathcal{F}_{\text {sun }}(t)=S_{0}(1-A)(1-C) \mathcal{H}(t)
$$

to approximate the short-wavelength solar flux. In Eq. (3) $S_{0}=1353\left[\mathrm{~W} / \mathrm{m}^{2}\right]$ is the solar constant, $A$ is the ground albedo, $C$ is a factor that accounts for the reduction in solar flux due to cloud cover, and $\mathcal{H}(t)$ is the local insolation function. Explicit expressions for the local insolation $\mathcal{H}(t)$ can be found in the literature. ${ }^{13,22}$ The quantity $\mathcal{F}_{s k y}$ is the long-wavelength radiation from the atmosphere given by Stefan's law, $\mathcal{F}_{s k y}(t)=\sigma T_{s k y}^{4}(t)$

\footnotetext{
${ }^{*}$ In formulating this expression we have ignored changes in the state in the medium, which would appear as a latent heat term. Such terms have been considered previously by Kahle ${ }^{15}$ and England. ${ }^{21}$
} 
where $\sigma=5.67 \times 10^{-8}\left[\mathrm{~W} \mathrm{~m}^{-2} \mathrm{~K}^{-4}\right]$ is the Stephen-Boltzman constant and $T_{s k y}[\mathrm{~K}]$ is an effective sky radiance temperature. The heat loss due to ground radiation is given by a similar expression, $\mathcal{F}_{g r}(t)=\mathcal{E} \sigma T_{g r}^{4}(t)$ where $\mathcal{E}$ is the mean emissivity of the surface and $T_{g r}(t)$ is the soil temperature at the soil-air interface. Kahle ${ }^{15}$ modeled sensible heat transfer between the surface and atmosphere as

$$
\mathcal{F}_{s h}(t)=\rho_{a} c_{p a} C_{d}(W(t)+2)\left(T_{a i r}(t)-T_{g r}(t)\right) .
$$

where $\rho_{a}=1.16\left[\mathrm{~kg} / \mathrm{m}^{3}\right]$ is the density of air, $c_{p a}=1007\left[\mathrm{~J} \mathrm{~kg}^{-1} \mathrm{~K}^{-1}\right]$ is the specific heat of air, $C_{d}$ is the wind drag coefficient (chosen to be 0.002), and $W(t)$ is the wind speed. Kahle, ${ }^{15}$ drawing on measurements by Kondratyev, ${ }^{23}$ formulated an empirical model for the air temperature, which was later modified by England ${ }^{17}$ to yield the expression

$$
T_{\text {air }}(t)=T_{0, a i r}-T_{\text {del }} \cos (2 \pi(t-2) / 24),
$$

where $t$ is the local time (in hours) measured from midnight, and $T_{0, a i r}$ and $T_{\text {del }}$ are estimated from local meteorological data available from the National Weather Service. Kahle also proposed a model for the sky temperature, which was modified by England ${ }^{17}$ using Brundt's formula ${ }^{15}$

$$
T_{\text {sky }}(t)=T_{\text {air }}(t)(0.61+0.05 \sqrt{w})^{0.25},
$$

where $w$ is the water vapor pressure in mmHg.

The radiative components seen by the IR camera include (1) thermal radiation from the soil surface, (2) soilreflected sunlight, (3) soil-reflected skylight (thermal emission from the atmosphere and sunlight scattered by particles and air molecules), and (4) a negligible amount of thermal emission from the atmosphere directly to the camera. Thermal emission from the soil surface depends on the surface temperature and emissivity. The surface temperature distribution can be obtained from the solution of Eq. (1) with the boundary condition given by Eq. (2). The power emitted by the soil surface is determined from the soil's spectral radiance function. The radiance function for that graybody emitter is given by the product of the surface emissivity $\mathcal{E}$ and the blackbody spectral radiance $L_{\lambda}(T)$ described by Planck's radiation law

$$
L_{\lambda}(T)=\frac{2 c^{2} h}{\lambda^{5}} \frac{1}{e^{h \nu / k T}-1} \quad\left[\mathrm{~W} \mathrm{~m}{ }^{-2} \mathrm{sr}^{-1} \mu \mathrm{m}^{-1}\right]
$$

where $h=6.63 \times 10^{-34}[\mathrm{~J} \mathrm{~s}]$ is Planck's constant, $\nu[\mathrm{Hz}]$ is the frequency of the optical radiation, $\lambda[\mathrm{m}]$ is the wavelength of that radiation, $k=1.38 \times 10^{-23}\left[\mathrm{~J} \mathrm{~K}^{-1}\right]$ is Boltzmann's constant, and $c$ is the speed of light $\left[\mathrm{m} \mathrm{s}^{-1}\right]$. We write the radiation emitted by the surface as the product

$$
L_{\text {surf }}(\lambda, \theta, \phi, \mathbf{r})=\mathcal{E}(\lambda, \theta, \phi, \mathbf{r}) L_{\lambda}(T(\mathbf{r})),
$$

where $\mathcal{E}(\lambda, \theta, \phi, \mathbf{r})$ is the spectral directional emissivity. In this work we make the approximation that soil is a diffuse emitter, i.e., the emissivity is independent of direction.

From the above expressions it is clear that emissivity has a direct effect on the thermal signature. In addition, grass and other forms of ground cover have a strong effect on the heat flow process and on the reflection and radiation of thermal energy. A study of the emissivity of natural materials has been made by Salisbury and D'Aria. ${ }^{24,25}$ The reflectivity of these materials can be obtained from the emissivity values by using the Kirchoff's law for an opaque body, $\mathcal{R}=1-\mathcal{E}$. Using the diffuse emitter approximation, the received radiance can be written as

$$
L_{r e c}(\lambda, \mathbf{r})=\mathcal{E}(\lambda, \mathbf{r}) L_{\text {surf }}(\lambda, \mathbf{r})+\mathcal{R}(\lambda, \mathbf{r})\left[L_{\text {sun }}(\lambda)+L_{\text {sky }}(\lambda)\right]
$$

This radiance expression permits us to calculate the power incident on an IR detector surface $D$ from the radiating soil surface $S$. The power incident on the detector can be found by

$$
\Phi=\int_{\lambda_{1}}^{\lambda_{2}} d \lambda \int_{D} d D \int_{S} d S L_{r e c}(\lambda, \mathbf{r}) \frac{\cos \theta_{1} \cos \theta_{2}}{R^{2}}
$$

where $R$ is the distance between a point on surface $S$ and a point on the detector $D, \cos \theta_{1}$ and $\cos \theta_{2}$ are the projection of normal vectors for the surfaces $D$ and $S$ in the direction of the radiation, and the spectral band of interest is $\lambda_{1}-\lambda_{2}$. The IR image is formed by Eq. (10). 


\section{THERMAL MODELING USING THE FINITE ELEMENT METHOD}

The finite element method (FEM) is an efficient computational technique, widely used for the solution of heat transfer problems. The essential feature of this technique is the spatial discretization of the computational domain. In what follows we denote the computational volume by $\Omega$ and its boundary by $\Gamma$. We take this volume to have a parallelepiped shape (with the exception of the rough surface at the soil-air interface). The boundary planes in $x$ and $y$ of the computational volume are denoted $\Gamma_{x}$ and $\Gamma_{y}$ respectively. The lower boundary plane is denoted $\Gamma_{z}$. An individual element of the subdivided volume and its boundary are represented by $\Omega_{e}$ and $\Gamma_{e}$, respectively. The element boundaries on the soil-air interface are represented with $\Gamma_{e, a i r}$.

We require the steady state solution of Eq. (1) with suitable boundary conditions when driven by a time-varying source. The temperature ranges of interest are relatively small (at most, a few tens of Kelvins), and over this limited range the thermal properties of soil and mine are assumed to be independent of temperature and of time. As a result, phenomena such as the drying of soil and the movement of soil water are neglected in the current model. We also assume that the temporal and spatial dependencies of $T(\mathbf{r}, t)$ are separable within an element.

Over each element $\Omega^{e}$ we approximate the temperature distribution as

$$
T^{e}(\mathbf{r}, t)=\sum_{i=1}^{N_{e}} T_{i}^{e}(t) \phi_{i}^{e}(\mathbf{r}),
$$

where $\phi_{i}^{e}(\mathbf{r})$ are user-prescribed interpolation functions, $N_{e}$ denotes the number of nodes over the subdivided finite element, and $T_{i}^{e}(t)$ are the nodal temperatures which comprise the unknown coefficients in our representation. Using this expression in Eq. (1) and enforcing weak equality with the weighting function $w(\mathbf{r})$ we obtain

$$
\int_{\Omega_{e}} w(\mathbf{r})\left[\rho(\mathbf{r}) C_{p}(\mathbf{r}) \frac{\partial T^{e}(\mathbf{r}, t)}{\partial t}-\nabla \cdot\left(\mathcal{K}(\mathbf{r}) \nabla T^{e}(\mathbf{r}, t)\right)\right] d \mathbf{r}=0 \quad \forall \Omega^{e} \in \Omega .
$$

Continuity in temperature and flux are enforced on the surface $\Gamma^{e}$ shared by adjacent elements. On $\Gamma$ external boundary conditions (described below) are imposed. We use the Galerkin formulation, in which the weighting functions are chosen to be identical to the interpolation functions. This formulation minimizes the mean error over the volume and is the best approximation in the variational sense.

The boundary conditions imposed on the problem are determined from physical considerations. For the transverse coordinates $x$ and $y$, we assume the presence of an infinite rectilinear array of mines (e.g., a mine field). As a result of symmetry arguments, we can set the normal derivatives of $T$ equal to zero on $\Gamma_{x}$ and $\Gamma_{y}$. Although an infinite array of mines is being analyzed, for a sufficiently large computational volume, there is negligible interaction between mines, and the results are a good approximation to the response of an isolated mine. For the boundary condition on $\Gamma_{z}$ (at the bottom face of the computational volume) we observe that at sufficiently large depths (a few tens of centimeters) the temperature is independent of time over a diurnal cycle. This typical "diurnal depth" can be predicted from one-dimensional models of soil ${ }^{19}$ using thermal characteristics found in the literature. ${ }^{26-28}$ On the basis of these findings, we can set $T$ equal to a constant on $\Gamma_{z}$.

It remains to specify the boundary condition at the soil-air interface. As noted in Eq. (2) this condition involves a nonlinear function of $T$. Over the limited temperature range of interest, however, the result can be linearized using a technique described by Watson. ${ }^{13}$ We write

$$
\frac{\partial T(\mathbf{r}, t)}{\partial z} \approx T(\mathbf{r}, t)\left(\rho_{a} c_{p a} C_{d}(W(t)+2)+4 \epsilon \sigma T_{s k y}^{3}(t)\right)-\mathcal{F}_{\text {sun }}(t)-\rho_{a} c_{p a} C_{d}(W(t)+2) T_{a i r}(t)-4 \epsilon \sigma T_{s k y}^{4}(t) .
$$

This change renders the entire problem linear.

A boundary condition at $t=0$ is also required. Although the solution procedure described below will converge to a steady state value using $T(\mathbf{r}, t=0)=0$ as the initial condition, the computation time can be reduced if the average depth-dependent temperature of homogeneous soil is used as a starting value. A suitable estimate is given by

$$
T(\mathbf{r}, t=0)=\frac{\frac{1}{L_{p}} \int_{L_{p}} \mathcal{F}_{\text {sun }}(t) d t+4 \epsilon \sigma \bar{T}_{s k y}^{4}+\rho_{a} c_{p a} C_{d}(\bar{W}+2) \bar{T}_{a i r}}{4 \epsilon \sigma \bar{T}_{s k y}^{3}+\rho_{a} c_{p a} C_{d}(\bar{W}+2)}
$$


In Eq. (14) $L_{p}$ denotes the 24 hour period and $\bar{T}_{s k y}, \bar{T}_{a i r}$, and $\bar{W}$ correspond to the average values of sky temperature, air temperature and wind speed, respectively, over this period. A similar expression neglecting the convection term was derived by Watson. ${ }^{13}$

After substituting Eq. (11) in Eq. (12), incorporating the boundary conditions and performing some mathematical manipulations, the unknown nodal coefficients $T_{i}(t)$ can be expressed as a matrix equation

$$
\overline{\mathbf{M}} \dot{\mathbf{T}}+\overline{\mathbf{K}} \mathbf{T}=\mathbf{F},
$$

in which boldface letters represent vectors, overlined boldface letters represent matrices and the superposed dot on $\mathbf{T}$ denotes the derivative with respect to time. The elements of these matrices and vectors are given by

$$
\begin{gathered}
M_{i j}=\int_{\Omega_{e}} \rho(\mathbf{r}) C_{p}(\mathbf{r}) \phi_{i}^{e}(\mathbf{r}) \phi_{j}^{e}(\mathbf{r}) d \mathbf{r} \\
K_{i j}=\int_{\Omega_{e}} \mathcal{K}(\mathbf{r})\left[\frac{\partial \phi_{i}^{e}(\mathbf{r})}{\partial x} \frac{\partial \phi_{j}^{e}(\mathbf{r})}{\partial x}+\frac{\partial \phi_{i}^{e}(\mathbf{r})}{\partial y} \frac{\partial \phi_{j}^{e}(\mathbf{r})}{\partial y}+\frac{\partial \phi_{i}^{e}(\mathbf{r})}{\partial z} \frac{\partial \phi_{j}^{e}(\mathbf{r})}{\partial z}\right] d \mathbf{r} \\
+\int_{\Gamma_{e, a i r}}\left[4 \epsilon \sigma T_{s k y}^{3}(t)+\rho_{a} c_{p a} C_{d}(W(t)+2)\right] \phi_{i}^{e}(\mathbf{r}) \phi_{j}^{e}(\mathbf{r}) d S
\end{gathered}
$$

and

$$
F_{i}=\int_{\Gamma_{e, a i r}}\left[4 \epsilon \sigma T_{s k y}^{4}(t)+\rho_{a} c_{p a} C_{d}(W(t)+2) \sigma T_{a i r}(t)+\mathcal{F}_{\text {sun }}(t)\right] \phi_{i}^{e}(\mathbf{r}) d S .
$$

The spatial discretization was accomplished using pentahedral elements. A sample spatial discretization is shown in Fig. 1. This choice of element allows us to represent an arbitrary rough surface and it is amenable to the calculations required in our radiometric model. Linear interpolation functions $\phi_{i}^{e}(\mathbf{r})$ were chosen.

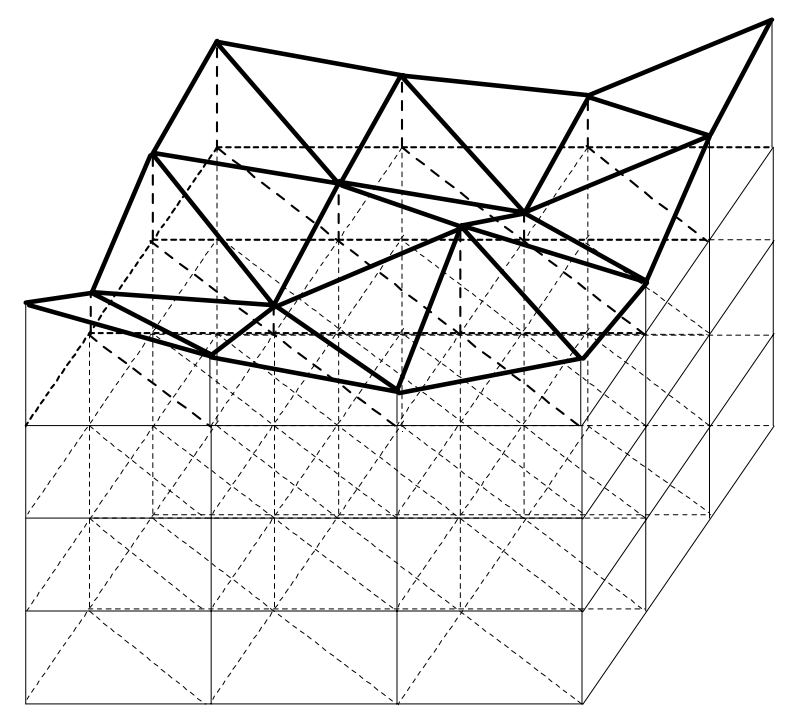

Figure 1. Sample spatial discretization of the computational domain. The volume is subdivided into pentahedral elements resulting in a triangular model for the soil surface.

Temporal discretization of Eq. (15) leads to a finite difference formulation, which is solved using the CrankNicholson scheme. We have

$$
\left(2 \overline{\mathbf{M}}+\Delta t \overline{\mathbf{K}}_{t+\Delta t}\right) \mathbf{T}_{t+\Delta t}=\Delta t\left(\mathbf{F}+\mathbf{F}_{t+\Delta t}\right)+\left(2 \overline{\mathbf{M}}+\Delta t \overline{\mathbf{K}}_{t}\right) \mathbf{T}_{t}
$$

Eq. (19) is solved at each time step to evaluate the nodal temperatures $\mathbf{T}_{t+\Delta t}$. Employing an LU decomposition technique for this solution is inefficient because the matrices $\overline{\mathbf{M}}$ and $\overline{\mathbf{K}}$ are sparse. Banded matrix storage provides a partial remedy for this problem, but limitations in node numbering usually results in ineffecient memory use. An efficient sparse solver is employed in our work. 


\section{RADIOMETRIC MODELING}

To predict the mine signatures seen by an IR camera, a radiometric model must be combined with the thermal FEM model. The radiometric model describes the spatial and spectral characteristics of the fluence transmitted from the soil surface to the IR detector. The model requires the spectral behaviour of the source (sunlight and skylight), a rough surface model to describe the soil-air interface, and the spectral behaviour of the soil. A flat spectral model is assumed for soil in this paper. A more realistic model is being investigated.

\subsection{Sunlight and Skylight Models}

The radiance due to sunlight and skylight comprise the source function for our themal model, and reflections from the soil are a radiometric component of interest. The total radiation over the entire spectrum due to sunlight and skylight are required for the thermal model and are given by $\mathcal{F}_{\text {sun }}$ and $\mathcal{F}_{\text {sky }}$, respectively. More detailed spectral radiance models are required to describe the reflected components seen by the IR camera.

The radiance $L_{\text {sun }}$ can be modeled with reasonable accuracy by a blackbody radiator. Neckel and Labs ${ }^{29}$ show that a blackbody source at $\approx 5800 \mathrm{~K}$ represents a good numerical fit to exoatmospheric measured radiance data in the visible and near IR bands. Thekaekara and Drummond ${ }^{30}$ summarized data regarding the components of the total radiation and their spectral distribution, and they proposed standard values for them. Measured solar radiation at different sites and models for meteorological and climatic factors are presented in a summary by Dogniaux ${ }^{31}$ prepared for the European Community Programme on Solar Energy. The measurement techniques and instruments are described in Coulson. ${ }^{32}$ Our radiometric model of $L_{\text {sun }}$ comprises the blackbody source approximation mentioned above. Atmospheric transmittance is included in our model using the data given by Allen $^{33}$ and approximated in a functional form by Watson. ${ }^{13}$

The quantity $L_{s k y}$ depends on the composition of the local atmosphere and is more difficult to model accurately. It comprises wide-angle Rayleigh scattering by molecular constituents (very weak at IR wavelengths), small-angle Mie scattering by aerosols, and thermal radiation from the warm atmosphere. The thermal contribution is most important at long wavelengths, and can be approximated by a blackbody radiator at the local air temperature. Although there has been extensive work in developing models for sunlight and skylight, local changes in atmospheric particulates and water vapor content can greatly affect model accuracy. In addition, a number of molecular species $\left(\mathrm{H}_{2} \mathrm{O}, \mathrm{CO}_{2}\right.$, $\mathrm{CO}, \mathrm{N}_{2} \mathrm{O}, \mathrm{O}_{3}$, and $\mathrm{CH}_{4}$ ) have absorption bands in the infrared, which affect the incident solar radiation. ${ }^{26}$ Our model for $L_{s k y}$ comprises a blackbody approximation at the local air temperature.

\subsection{Rough Surface Approximation}

Thermal emission and reflection of ambient light from a rough surface are of considerable importance in predicting the signature seen by an IR sensor. Variations in the surface normal direction affect the natural solar insolation falling on the surface, which influences both thermal heating and surface reflections. IR imagery of rough surfaces may also contain self-shadowing of the surface as a result of the topology. To address these effects we incorporated surface roughness into our thermal and radiometric models.

Rough surfaces were constructed by specifying random elevations for points in a rectilinear grid defined by the top surface of our FEM computational volume. The surface is then represented by fitting triangles to the grid points, as shown in Fig. 1. The same representation is used by both the thermal and radiometric models, which permits the modeling work to be decoupled to a degree. Predicting IR imagery over a rough soil surface is a computationally expensive process, because of the visible surface determination problem. Techniques devised for computer graphics ${ }^{34}$ offer significant reductions in the computational complexity.

Consider now the problem of generating a rough surface with a random height profile $z=f(x, y)$ having zero mean. For a Gaussian distributed surface this problem can be addressed by expressing the surface in the spatial spectral domain $\left(k_{x}, k_{y}\right)$. The spectrum $W\left(k_{x}, k_{y}\right)$ (or equivalently, the covariance or structure function) of the surface is assumed known. We also assumed a Gaussian form for the spectrum, namely:

$$
W\left(k_{x}, k_{y}\right)=\frac{L_{x} L_{y} h^{2}}{4 \pi} \exp \left(-\frac{L_{x}^{2} k_{x}^{2}}{4}-\frac{L_{y}^{2} k_{y}^{2}}{4}\right),
$$

where $L_{x}$ and $L_{y}$ are correlation lengths in the $x$ and $y$ directions respectively, and $h$ is the surface rms height. In what follows we take $L_{x}=L_{y}=L$. The image is formed via an inverse Fourier transform of a matrix of complex 
random numbers with uniformly distributed phase and amplitude $\sqrt{W\left(k_{x}, k_{y}\right)}$. Some examples of random surfaces generated in this manner are shown in Fig. 2.

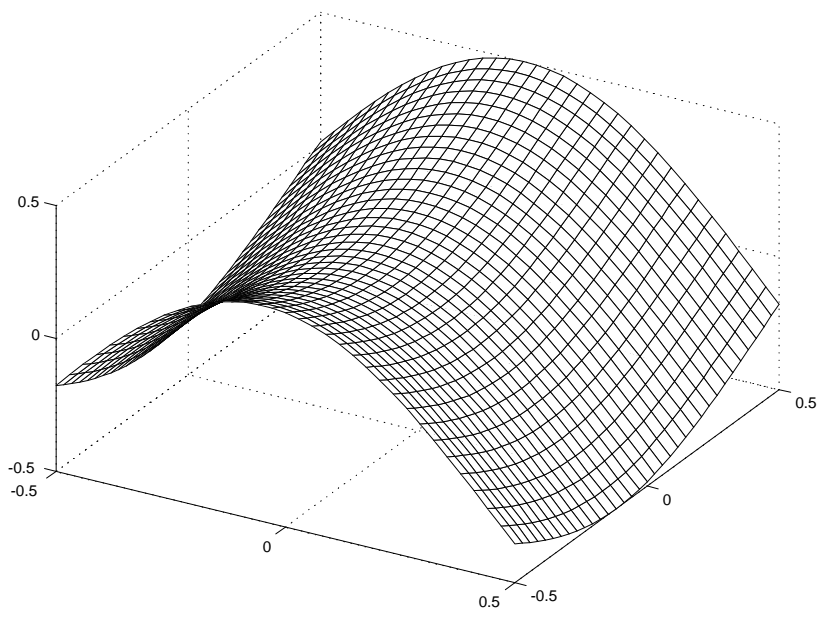

(a) $L=100 \mathrm{~cm}$.

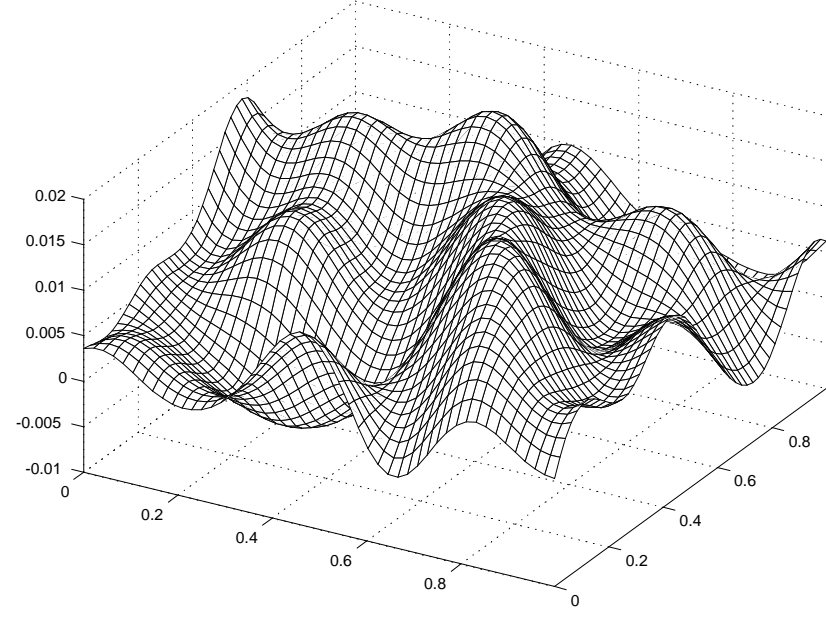

(b) $L=20 \mathrm{~cm}$

Figure 2. Examples of rough surfaces realized using for a Gaussian spectrum.

\section{RESULTS}

The models described above were used to simulate the temporal and spatial signatures of a mine buried under smooth and rough surfaces. In all simulations a steady wind speed of $W(t)=\bar{W}=2\left[\mathrm{~m} \mathrm{~s}^{-1}\right]$ and an average air temperature of $\bar{T}_{\text {air }}=289 \mathrm{~K}$ were used. The thermal diffusivity $(\kappa)$ and conductivity $(\mathcal{K})$ of soil were taken to be $5.0 \times 10^{-7}\left[\mathrm{~m}^{2}\right.$ $\left.\mathrm{s}^{-1}\right]$ and $2.6\left[\mathrm{~W} \mathrm{~m}^{-1} \mathrm{~K}^{-1}\right]$, respectively. For TNT we used $\kappa=9.25 \times 10^{-8}\left[\mathrm{~m}^{2} \mathrm{~s}^{-1}\right]$ and $\mathcal{K}=0.234\left[\mathrm{~W} \mathrm{~m}^{-1} \mathrm{~K}^{-1}\right]$. It is noteworthy that TNT is a better thermal insulator than soil. Mine dimensions and construction details vary significantly. As a representative target, we selected a simulant anti-tank mine ${ }^{35}$ developed by the US Army. The simulant mine has a diameter of $25 \mathrm{~cm}$ and a height of $8.33 \mathrm{~cm}$. The mine is assumed to be composed of TNT as shown in Fig. 3. ${ }^{\dagger}$

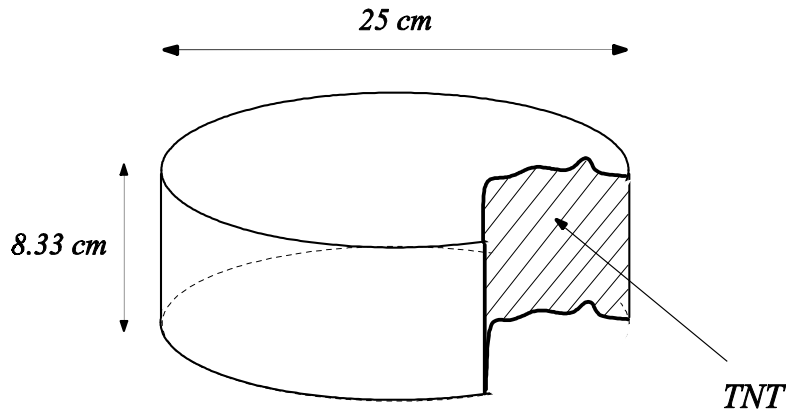

Figure 3. Model of the simulant anti-tank mine used in the simulations.

The computational volume has the dimensions $0.9984 \times 0.9984 \times 0.3154 \mathrm{~m}^{3}$ and was discretized with pentahedral elements of size $2.08 \times 2.08 \times 1.66 \mathrm{~cm}^{3}$ in the $x, y$, and $z$ directions, respectively. This discretization results in 87552 finite elements and 48020 nodes, which also determines the size of the resulting matrix equation. The memory requirement of the FEM code for this discretization is about $250 \mathrm{MB}$. The Crank-Nicholson scheme is unconditionally stable, which permits us substantial freedom in choosing the time increment. The time increment was selected to

\footnotetext{
†The anti-tank simulants contain small metal inserts located at the center of the bottom face of the mine. This insert was not modeled in our work. We do not expect to incur a significant error as a result, because of the insert's size and location.
} 
be 360 seconds, which gives good resolution over a 24 hour period. The FEM solution was observed to reach steady after only two simulated diurnal cycles. The simulation takes about 2 hours on a $300 \mathrm{MHz}$ Pentium II machine.

The radiometric model requires the triangular rough surface representation and the output of the thermal model. Using these data and the geometry given in Fig. 4, we construct a virtual IR camera. The virtual sensor is assumed to be a LWIR camera operating near $10 \mu \mathrm{m}$. A pixel array of 160 (horizontally) by 120 (vertically) is assumed with an instantaneous field of view of 1 mrad.

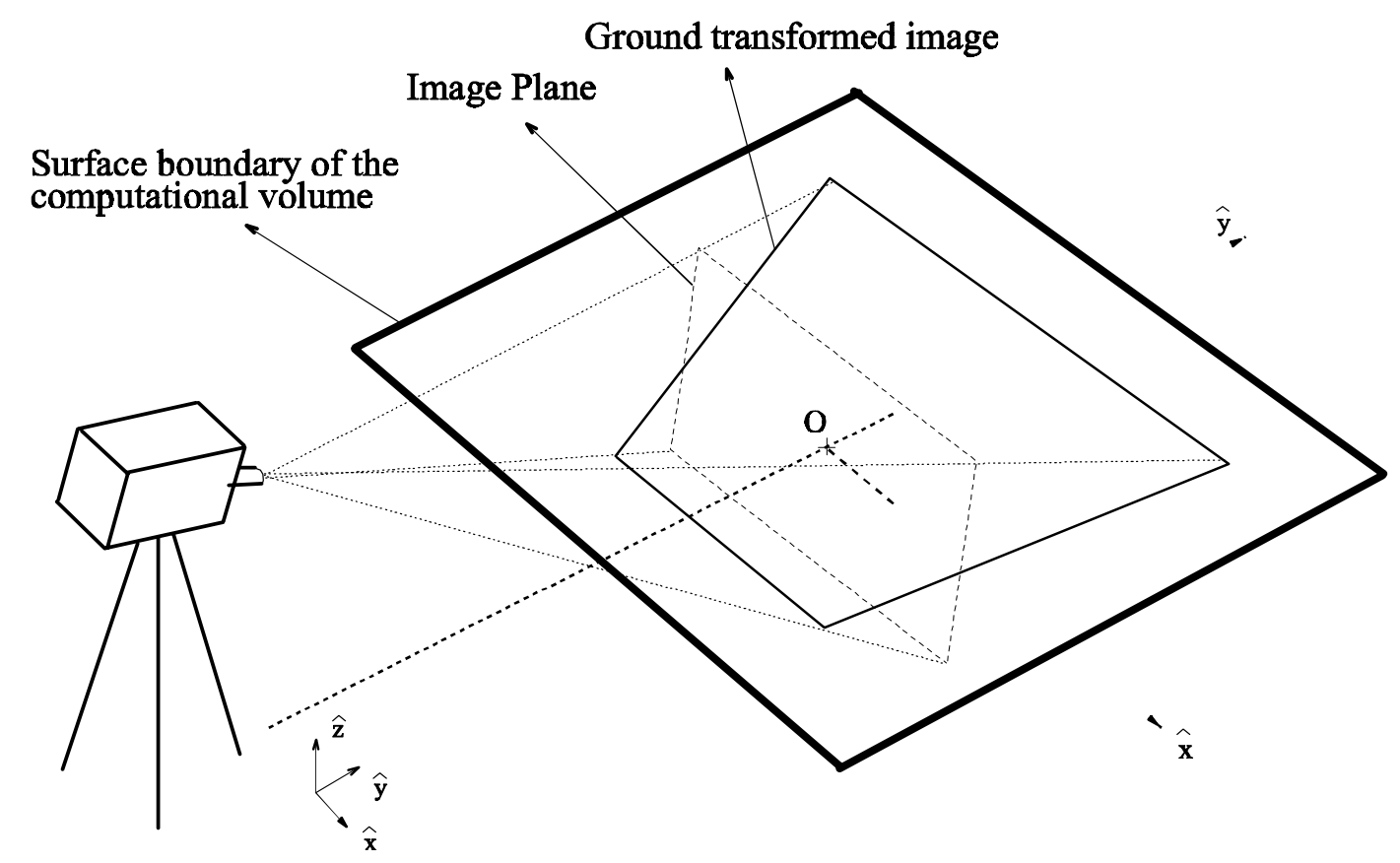

Figure 4. Sketch of the region of interest including the IR camera. Point $O$ is both the center of the field of view and the origin of coordinates. The image plane, the ground transformed image, the surface boundaries of the computational volume, and the global axis are illustrated.

The IR camera is aimed at point $\mathrm{O}$ as shown in Fig. 4. Point $\mathrm{O}$ is also the global origin for both the thermal and radiometric models. To cover the surface above the mine with sufficient resolution, an appropriate camera locations and height must be identified. For this work we selected a camera vertical height of 2 meters, and a horizontal standoff (camera center to point $\mathrm{O}$ ) of 3 meters. The resulting ground-projected field of view encompasses most of the computational volume.

Figure 5 shows the surface temperature distribution over the mine. The results are presented as a sequence of images evaluated at three hour time increments starting from sunrise. It is experimentally observed that contrast changes occur twice daily at the thermal cross-over times, and these events appear in our simulations. The results suggest that the physical temperature differences during the day peak at roughly $1.73 \mathrm{~K}$ occuring about 2 hours before sunset. The temperature difference has a maximum at the center of the mine and diminishes as we move away from the center. The temperature distribution as a function of depth is presented in Fig. 6 . The location of the mine is emphasized with a rectangle. The temperature distribution is again given every three hours starting at sunrise. The figures present the evolution of heat flow into the ground and the influence of the mine on this heat flow. The surface temperature over the mine is cooler at dawn, and it warms as time proceeds. The mine tend to block the flow of heat into the soil, causing the overlying soil to become hotter during the day. During the night, the mine blocks the upward flow of energy in the soil, permitting the layer of soil above the mine to cool more rapidly. These observations and our previous findings using one-dimensional models ${ }^{19}$ are consistent with the behavior of insulating mines.

Simulations of the $25 \mathrm{~cm}$ anti-tank mine simulant appear in Figs. 7 and 8 for the case of a smooth surface and a surface having a $3 \mathrm{~cm}$ peak-to-peak variation. The rough surface decorrelation length was $20 \mathrm{~cm}$, which leads to reasonably large surface slopes. While the smooth surface case is substantially the same as a perspective-transformed 
copy of the thermal surface images shown in Fig. 5, the rough surface case shows significant clutter-like variations, which could easily be mis-detected as false alarms.

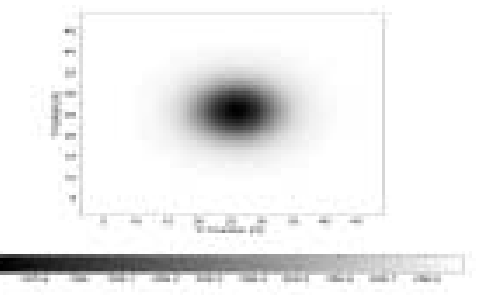

(a)

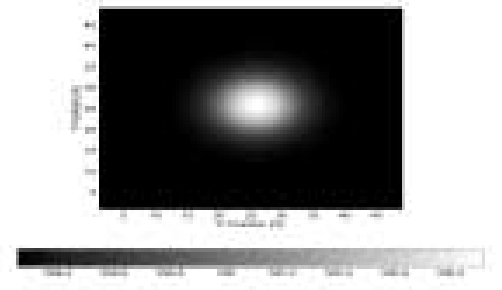

(d)

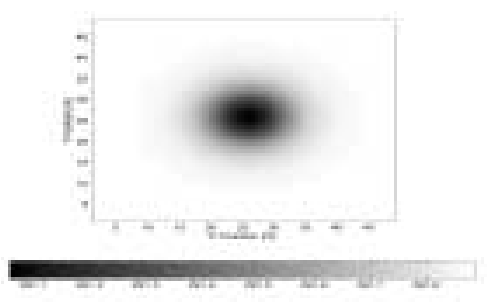

(b)

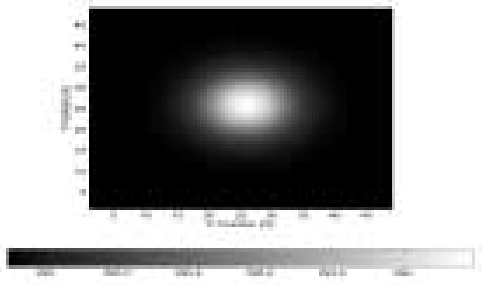

(e)

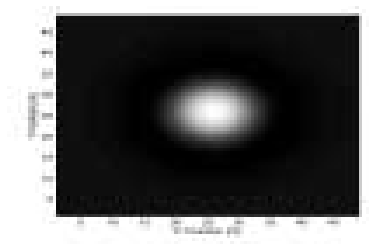

(c)

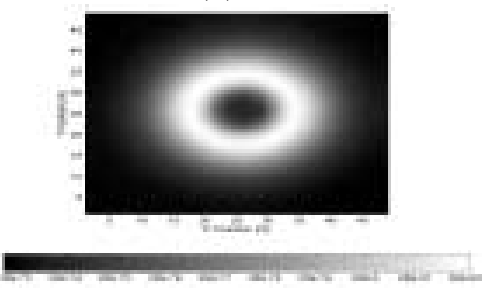

(f)

Figure 5. Simulation of the soil surface temperature difference over the anti-tank mine simulant buried $6.66 \mathrm{~cm}$ under a smooth soil surface. The temperature distribution is evaluated at different times of the day (a) At dawn. (b) 3 hours after sunrise. (c) At noon. (d) 3 hours after noon. (e) At sunset. (f) 3 hours after sunset.

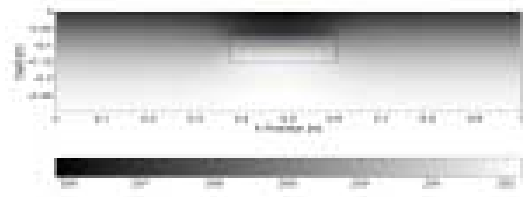

(a)

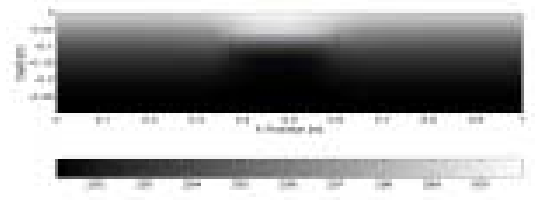

(d)

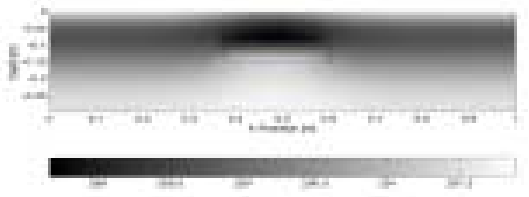

(b)

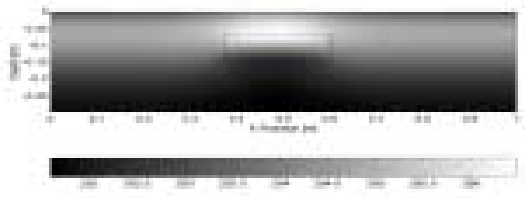

(e)

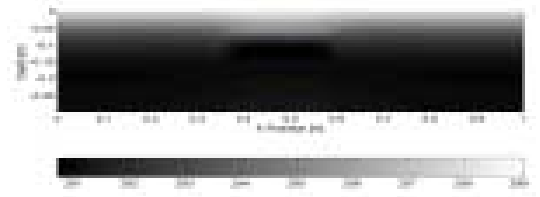

(c)

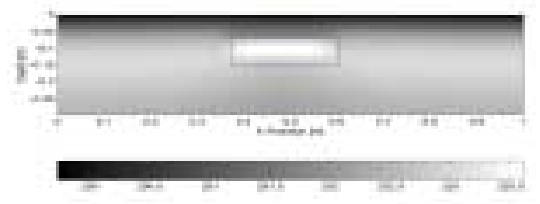

(f)

Figure 6. Simulation of the temperature distribution at depth taken through the middle of the anti-tank mine simulant buried $6.66 \mathrm{~cm}$ under a smooth soil surface. The temperature distribution is evaluated at different times of the day (a) At dawn. (b) 3 hours after sunrise. (c) At noon. (d) 3 hours after noon. (e) At sunset. (f) 3 hours after sunset. 


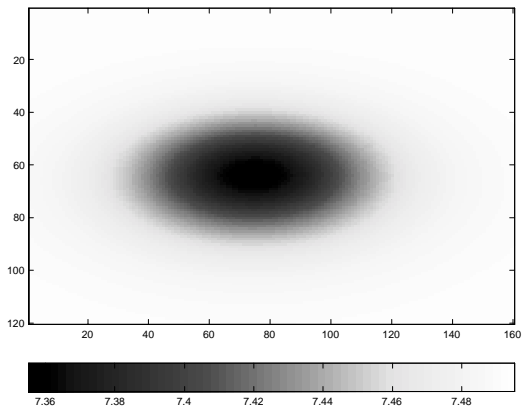

(a)

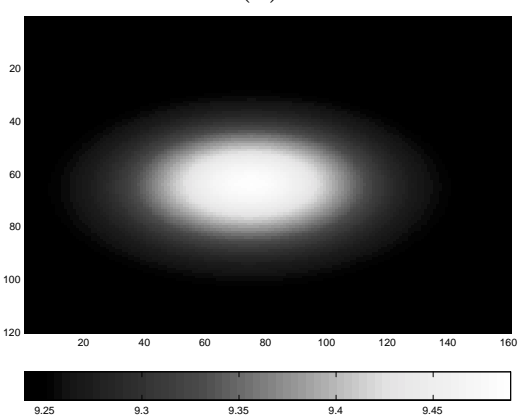

(d)
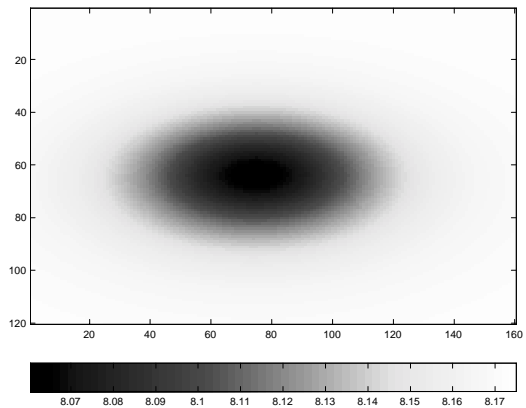

(b)

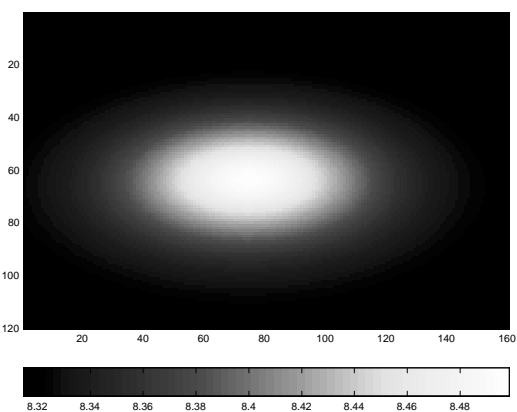

(e)
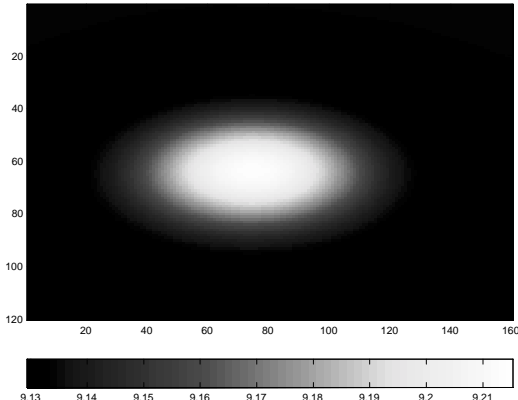

(c)

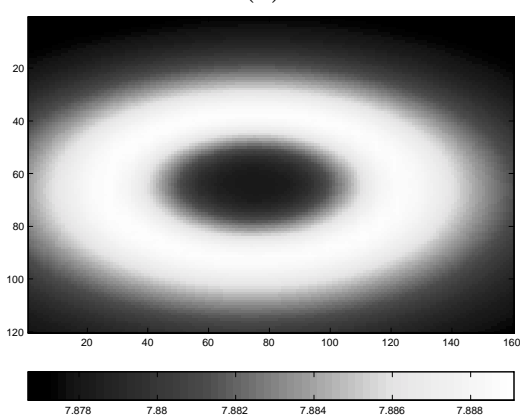

(f)

Figure 7. Simulation of the IR camera response for the SIM-25 mine buried $6.66 \mathrm{~cm}$ under a smooth soil surface. The radiometric model is used to predict the IR camera response at different times of the day (a) At dawn. (b) 3 hours after sunrise. (c) At noon. (d) 3 hours after noon. (e) At sunset. (f) 3 hours after sunset.

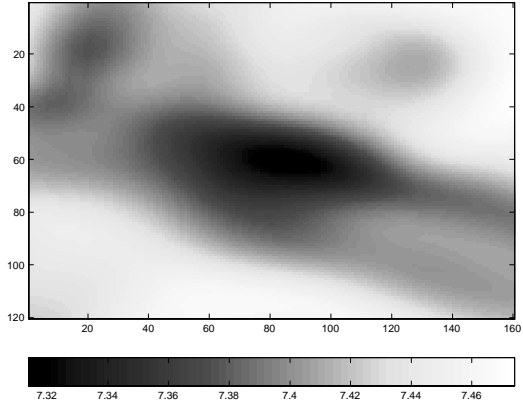

(a)

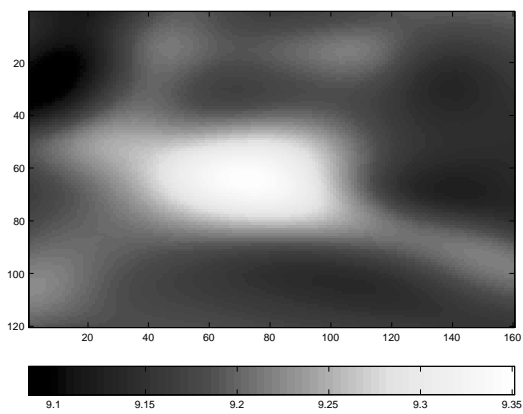

(d)

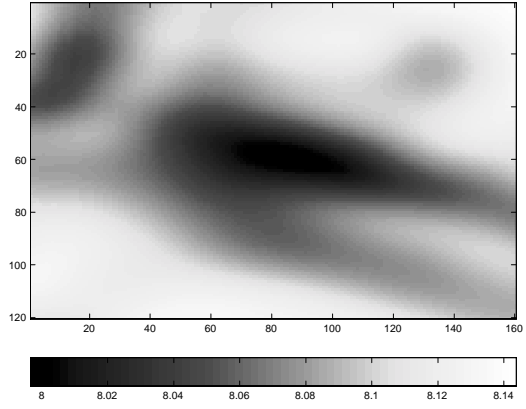

(b)

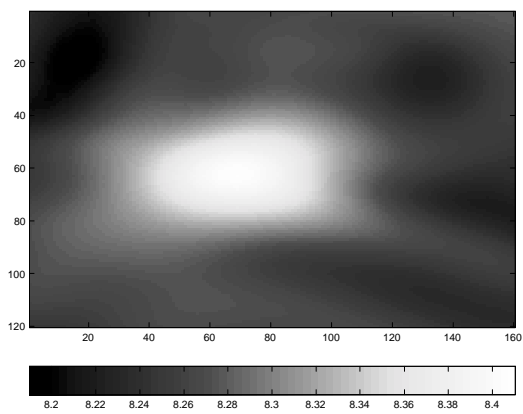

(e)

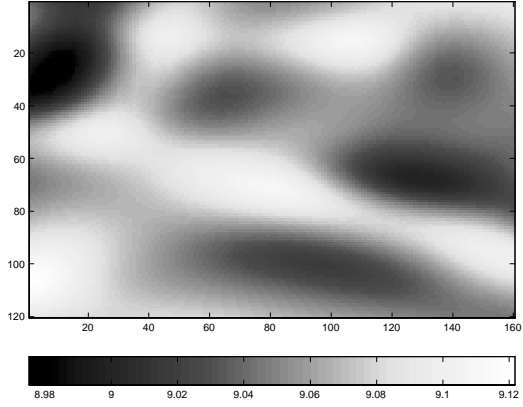

(c)

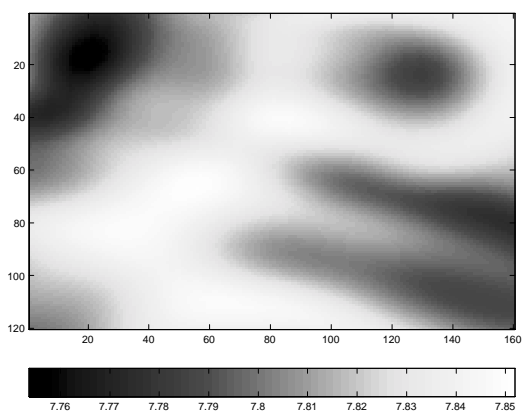

(f)

Figure 8. Simulation of the IR camera response for the SIM-25 mine buried $6.66 \mathrm{~cm}$ under a rough soil surface with peak-to-peak height $3 \mathrm{~cm}$. The radiometric model is used to predict the IR camera response at different times of the day (a) At dawn. (b) 3 hours after sunrise. (c) At noon. (d) 3 hours after noon. (e) At sunset. (f) 3 hours after sunset. 


\section{SUMMARY AND CONCLUDING REMARKS}

A model for thermal and radiometric effects has recently been developed for predicting the IR signature of buried land mines. The model employs a 3-D numerical solution of the thermal problem based on the finite element method (FEM). This approach permits inhomogeneous soil and the internal structure of the mine to be considered in modeling, although we have not exercised those capabilities in this initial study. In addition, the model incorporates a rough surface for the soil-air interface, which has implications for both thermal heating and for reflected radiometric terms. The temperature distribution computed using the thermal model is combined with surface-reflected radiometric components to produce the image seen at a virtual IR sensor.

Several extensions of this work are called for. Example calculations were presented, which show trends observed experimentally, but careful experimental validation of the code is necessary. The spectral content of natural sources and the spectral dependence of nature materials were largely ignored in this work, but will be addressed. Soil inhomogeneity has not been considered, but it presents no significant challenges to our formulation. A Gaussian surface model was assumed. We plan to acquire more realistic surface models using a laser surface profiler, and we intend to incorporate such data in a future work. Surface mines present additional challenges, which must also be addressed.

\section{ACKNOWLEDGMENTS}

This project was supported by funds from Duke University under an award from the ARO (the OSD MURI program). The findings, opinions and recommendations expressed therein are those of the author and are not necessarily those of Duke University or the ARO.

\section{REFERENCES}

1. L. A. LeSchack and N. K. Del Grande, "A dual-wavelength thermal infrared scanner as a potential airborne geophysical exploration tool," Geophys. 41(6), pp. p. 1318-1336, 1976.

2. Y. H. L. Janssen, A. N. de Jong, H. Winkel, and F. J. M. van Putten, "Detection of surface laid and buried mines with IR and CCD cameras, an evaluation based on measurements," in Detection and Remediation Technologies for Mines and Minelike Targets, A. C. Dubey, R. L. Barnard, C. J. Lowe, and J. E. McFee, eds., Proceedings of SPIE 2765, pp. 448-459, 1996.

3. G. Maksymomko, B. Ware, and D. Poole, "A characterization of diurnal and environmental effects on mines and the factors influencing the performance of mine detecting ATR algorithms," in Detection and Remediation Technologies for Mines and Minelike Targets, A. C. Dubey, I. Cindrich, J. M. Ralston, and K. Rigano, eds., Proceedings of SPIE 2496, pp. 140-151, 1995.

4. J. R. Simard, "Improved lanmine detection capability(ILDC): Systematic approach to the detection of buried mines using passive IR imaging," in Detection and Remediation Technologies for Mines and Minelike Targets, A. C. Dubey, R. L. Barnard, C. J. Lowe, and J. E. McFee, eds., Proceedings of SPIE 2765, pp. 489-500, 1996.

5. K. Russel, J. McFee, and W. Sirovyak, "Remote performance prediction for infrared imaging of buried mines," in Detection and Remediation Technologies for Mines and Minelike Targets II, A. C. Dubey and R. L. Barnard, eds., Proceedings of SPIE 3079, pp. 762-769, 1997.

6. B. A. Barbour, M. W. Jones, H. B. Barnes, and C. P. Lewis, "Passive IR polarization sensors: A new technology for mine detection," in Detection and Remediation Technologies for Mines and Minelike Targets III, A. C. Dubey, J. F. Harvey, and J. T. Broach, eds., Proceedings of SPIE 3392, pp. 96-103, 1996.

7. M. Larive, L. Collot, S. Breugnot, H. Botma, and P. Roos, "Laid and flush-buried mines (sic) detection using 8-12 $\mu \mathrm{m}$ polarimetric imager," in Detection and Remediation Technologies for Mines and Minelike Targets III, A. C. Dubey, J. F. Harvey, and J. T. Broach, eds., Proceedings of SPIE 3392, pp. 115-120, 1996.

8. C. DiMarzio, T. Vo-Dinh, and H. E. Scott, "Some approaches to infrared spectroscopy for detection of buried objects," in Detection and Remediation Technologies for Mines and Minelike Targets III, A. C. Dubey, J. F. Harvey, and J. T. Broach, eds., Proceedings of SPIE 3392, pp. 158-166, 1996.

9. P. Pregowski and W. Swiderski, "Using of (sic) comparison method in IR thermal detection of buried mines," in Detection and Remediation Technologies for Mines and Minelike Targets III, A. C. Dubey, J. F. Harvey, and J. T. Broach, eds., Proceedings of SPIE 3392, pp. 1241-1248, 1996. 
10. N. K. Del Grande, K. W. Dolan, P. F. Durbin, M. R. Gorvad, B. T. Kornblum, D. E. Perkins, D. J. Schneberk, and A. B. Shapiro, "Three-dimensional thermal imaging of structural flaws by dual-band infrared computed tomography," in Underground and Obscured Object Imaging and Detection, N. K. Del Grande, I. Cindrich, and P. B. Johnson, eds., Proceedings of SPIE 1942, pp. 207-215, 1993.

11. A. T. DePersia, A. Bowman, P. Lucey, and E. M. Winter, "Phenomenology considerations for hyperspectral mine detection," in Detection and Remediation Technologies for Mines and Minelike Targets, A. C. Dubey, I. Cindrich, J. M. Ralston, and K. Rigano, eds., Proceedings of SPIE 2496, pp. 159-167, 1995.

12. D. S. Flynn, D. A. Vechinsk, B. T. Blume, A. C. Dubey, and N. H. Witherspoon, "Minefield image synthesis tool," in Detection and Remediation Technologies for Mines and Minelike Targets, A. C. Dubey, I. Cindrich, J. M. Ralston, and K. Rigano, eds., Proceedings of SPIE 2496, pp. 208-221, 1995.

13. K. Watson, "Geologic application of thermal infrared images," Proc. IEEE 63(1), pp. 128-137, Jan. 1975.

14. A. W. England, J. F. Galantowicz, and M. S. Schretter, "The radiobrightness thermal inertia measure of soil moisture," IEEE Trans. Geosci. Remote Sensing 30(1), pp. 132-139, Jan. 1992.

15. A. B. Kahle , "A simple thermal model of the earth's surface for geologic mapping by remote sensing," Journal of Geophysical Research 82, pp. 1673-1680, 1977.

16. K. Watson, "Periodic heating of a layer over a semi-infinite solid," Journal of Geophysical Research $\mathbf{7 8}(26)$, pp. 5904-5910, Sept. 1973.

17. A. W. England, "Radiobrightness of diurnally heated, freezing soil," IEEE Trans. Geosci. Remote Sensing 28(4), pp. 464-476, July 1990.

18. Y.-A. Liou and A. W. England, "A land-surface process/radiobrightness model with coupled heat and moisture transport for freezing soils," IEEE Trans. Geosci. Remote Sensing 36(2), pp. 669-677, March 1998.

19. I. K. Sendur and B. A. Baertlein, "Thermal analysis of IR signatures for buried land mines," Progress Report to US Army Research Office on Contract 97-SC-ARO-1015, The Ohio State University ElectroScience Laboratory, Columbus, OH, March 1999.

20. Y.-A. Liou and A. W. England, "A land-surface process/radiobrightness model with coupled heat and moisture transport in soil," IEEE Trans. Geosci. Remote Sensing 36(1), pp. 273-286, Jan. 1998.

21. Y.-A. Liou and A. W. England, "Annual temperature and radiobrightness signatures for bare soils," IEEE Trans. Geosci. Remote Sensing 34(4), pp. 981-990, July 1996.

22. B. A. Baertlein and I. K. Sendur, "Thermal infrared detection of buried land mines," Progress Report to US Army Research Office on Contract 97-SC-ARO-1015, The Ohio State University ElectroScience Laboratory, Columbus, OH, December 1997.

23. K. Ya. Kondratyev, Radiation in the Atmosphere, Academic Press, New York, NY, 1969.

24. J. W. Salisbury and D. M. D'Aria, "Emissivity of terrestrial materials in the 8-14 $\mu \mathrm{m}$ atmospheric window," Remote Sensing Environment 42, pp. 83-106, 1992.

25. J. W. Salisbury and D. M. D'Aria, "Emissivity of terrestrial materials in the 3-5 $\mu \mathrm{m}$ atmospheric window," Remote Sensing Environment 47, pp. 345-361, 1994.

26. L. W. Wolfe and G. J. Zissis, The Infrared Handbook, revised edition, Environmental Research Institute of Michigan (ERIM), Ann Arbor, MI, 1989.

27. W. M. Rohsensow, J. P. Hartnett, and E. N. G. (eds.), Handbook of Heat Transfer Fundementals, 2nd ed., McGraw Hill, New York, NY, 1985.

28. R. G. Reeves, A. Anson, and D. L. (eds.), Manual of Remote Sensing, 1st ed., American Society of Photogrammetry, Falls Church, VA, 1973.

29. H. Neckel and D. Labs, "The solar radiation between 3300 and 12500 å," Solar Phys. 90, pp. 205-258, 1984.

30. M. P. Thekaekara and A. J. Drummond, "Standard values for the solar constant and its spectral components," Nature Physical Science 229(4), pp. 6-9, 1971.

31. R. Dogniaux (ed.), Prediction of Solar Radiation in Areas with a Specific Microclimate, First edition, Kluwer Academic Publishers, Inc., The Netherlands, 1994.

32. K. L. Coulson, Solar and Terrestrial Radiation, First edition, Academic Press, Inc., New York, NY, 1975.

33. C. W. Allen, Astrophysical Quantities, First edition, The Athlone Press., London, England, 1963.

34. J. D. Foley, A. van Dam, S. K. Feiner, and J. F. Hughes, Computer Graphics, Addison-Wesley Publishing Company, Inc., 1990.

35. Anon., "Simulant mines (sims)," tech. report, US Army Countermine and Demolition (Countermine Division), Fort Belvoir, VA, October 211998. 